\title{
DUE CASI PARTICOLARI DI RI-ETIMOLOGIZZAZIONE IN SOFOCLE: ODISSEO ED AIACE
}

\author{
Giovanna BATtaglino
}

Università degli Studi di Salerno, Itália

Resumo. Dois casos particulares de re-etimologização em Sófocles: Odisseu e Ájax. Nesta contribuição, procurar-se-á demonstrar como Sófocles - bem consciente do étimo difundido, fixado pelo mythos e/ou pela precedente produção lírica - realizou, em dois casos particulares uma consciente re-etimologização, em função do desenvolvimento do próprio drama.

Palavras-chave. Sófocles, tragédia, Ájax, Odisseu, re-etimologização.

Schneidewin, ${ }^{1}$ Commentando Il Luogo sofocleo, ${ }^{2}$ Nel quale Aiace stesso riflette sulla ov̉oía del proprio nome, acutamente rileva che "die tragischen Dichter lieben dergleichen einreiche Ausdeutungen der Eigennamen". ${ }^{3}$ Schneidewin coglieva, dunque, una particolare attitudine dei poeti tragici, i quali avrebbero manifestato una vera e propria predilezione per la tendenza ad etimologizzare o ad interpretare i nomi propri dei loro eroi, ravvisando, in essi, il riflesso di una particolare attitudine e/o aspetto del loro carattere o, addirittura, una vera e propria anticipazione del loro destino (compendiato nell'icastico sintagma nomen omen). In verità, la riflessione dello Schneidewin andrebbe ampliata, poiché la tendenza a ricercare il significato intrinseco dei nomi propri sarebbe una tendenza pienamente poetica - id est, non esclusivamente tragica, ma già epica e lirica -, come

"Artigo recebido em 22.abr.2014 e aceito para publicação em 7.jul.2014.

${ }_{1}^{1}$ Schneidewin è autore di un commento, nato ad usum scholarum, ma, tuttora, di grande utilità. Il commento fu, poi, rivisto dal Nauck ed è, ancor oggi, ritenuto molto utile all'esegesi delle tragedie sofoclee. Esso non ha, nella sua finalità didattica, un limite, bensì un punto di forza: pur nel suo carattere condensato e sintetico, è ricco di riflessioni sia di carattere linguistico, retorico e stilistico, che di carattere contenutistico.

${ }^{2}$ Ad Soph. Aj. 430-433. (S.v. infra.)

${ }^{3}$ Schneidewin 1865: 64. 
notava già Fuochi, ${ }^{4}$ il quale, in un suo corposo articolo, è il primo a fornirci una vera e propria fenomenologia di tale attitudine poetica. La tendenza alla etimologizzazione dei nomi propri è ravvisabile già in Omero e concerne, non a caso, il nome del protagonista, Odisseo. A tal proposito, si consideri Hom. Od. 19.405-9:

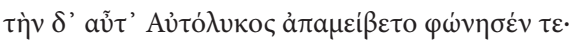

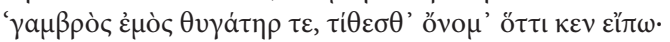

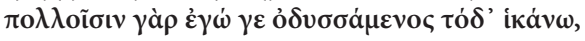

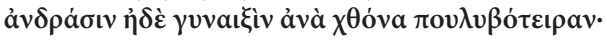

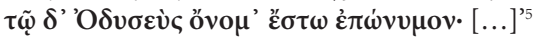

A lei [scil. ad Euriclea] rispondendo Autolico disse: "Genero mio e figlia mia, dategli il nome che io dico. Io sono qui giunto, avendo preso in odio molti uomini e donne sulla terra di molti uomini nutrice: dunque il nome che lo denomina sia Ulisse..." (trad. di Vincenzo Di Benedetto) ${ }^{6}$

Il passo omerico ivi citato rappresenta un contesto privilegiato, per quanto attiene all'etimologia dei nomi propri, dal momento che la spiegazione del nome proprio del protagonista è fornita esplicitamente ed è enfatizzata dal

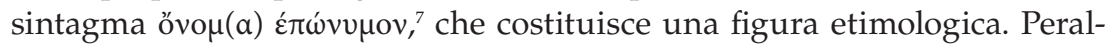
tro, ci troviamo in un contesto peculiare, in quanto il nome di Odisseo non gli deriva da vicende autobiografiche, né esprime il suo $\delta a i ́ \mu \omega v$, il suo destino - aliis verbis, non è connesso a nessuna delle sue avventure, le quali costituiscono l'oggetto del poema (come ci aspetteremmo) -, ma gli viene - per così dire - affibbiato dal nonno materno, sulla base della propria esperienza di vita. Così il nome di Odisseo appare quasi come un eteronimo. ${ }^{8}$

${ }^{4}$ Fuochi 1898: 273. Al Fuochi va il merito di aver dedicato un'ampia sezione di tale articolo all'individuazione degli strumenti, dei quali il poeta - il poeta tragico in particolare, ma non solopuò servirsi, per etimologizzare o interpretare un nome proprio. In sostanza, il poeta può ricorrere

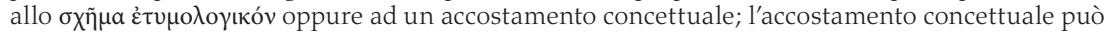
basarsi su un criterio di affinità oppure può essere antifrastico/ossimorico. Fuochi fornisce anche una vera e propria indagine sullo status quaestionis, menzionando gli studiosi che, prima di lui, si sono occupati del medesimo problema e discutendone criticamente l'approccio. Il tutto ha valore propedeutico-didascalico rispetto alla seconda - e più ampia - sezione del suo articolo, che ospita un vero e proprio elenco ragionato dei nomi propri che occorrono nelle tragedie di Eschilo, Sofocle ed Euripide. L'articolo di Fuochi, pur ben congegnato, manifesta evidenti limiti di natura "cronologica"; pertanto ho cercato di rimpinguarlo, con contributi più recenti.

${ }^{5}$ Per il testo omerico, seguo l'edizione critica di Allen (1908).

${ }^{6}$ Di Benedetto 2010: 1119.

${ }^{7}$ Esso, proprio a partire da Omero, si cristallizzerà, divenendo un sintagma topico, per quanto concerne l'introduzione dell'etimologia di un nome proprio.

${ }^{8}$ Utilizzo, qui - forse un po' impropriamente - il termine eteronimo, con valore di "nome attribuito da un altro". Per una trattazione approfondita del nome di Odisseo nell'Odissea, rimando al sapiente saggio di Miralles. Lo studioso analizza il problema del nome del protagonista dell'O- 


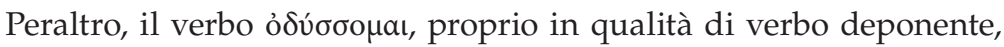
conserva uno statuto diatetico anfibologico. Di norma, tutti i commentatori

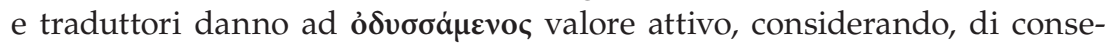

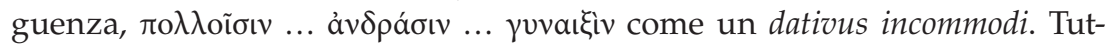
tavia, è plausibile che attribuire al verbo anche un valore passivo ("essere odiato da..."), attribuendo ai dativi appena menzionati un valore d'agente. Del resto, anche il LSJ ci conferma questa duplice valenza, dal momento che

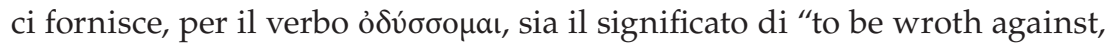
hate (with dative)", sia quello di "to be hated by (with dative)". Tra l'altro, la spiegazione del nome di Odisseo è fornita da un personaggio dotato, a sua volta, di un nome parlante: Autòlico vale, propriamente, "vero lupo" e, come notava Ferrari, si tratta di un nome "adatto a un tipico trickster, figlio di Hermes [...] e celebre per le sue frodi ed i suoi furti".

Il $\varphi \iota \lambda$ ó $\mu \eta \rho^{10}$ Sofocle dovette tener conto sia del contesto odissiaco,

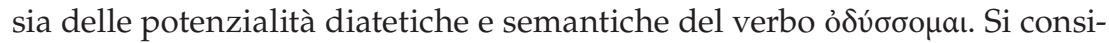
deri il fr. 965 R, tradito dalla Vita (20):

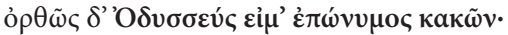

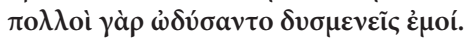

A buon diritto, ho un nome che trae origine dai mali: ${ }^{11}$ molti, infatti, mi odiarono, manifestando ostilità nei miei confronti. ${ }^{12}$

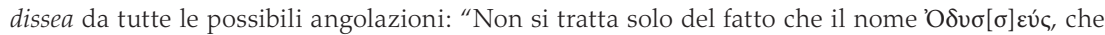
dà nome al poema, sia parlante. Ma anche che nel poema l'eroe stesso si dia un altro nome o altri parlino di lui, davanti a lui, e lo chiamino senza sapere chi sia o che egli si neghi o si proclami, o che il poeta ritardi nel nominarlo o lo nomini intenzionalmente in alcuni momenti e non in altri - $\mathrm{o}$ addirittura può accadere che dia per scontato che il suo pubblico ne conosca il nome che egli

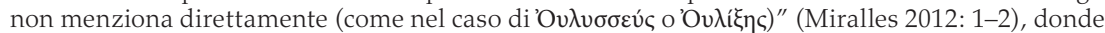
il latino Ulixes e l'italiano Ulisse. S.v. anche B. Marzullo, 1970, Il problema omerico, Milano-Napoli, Ricciardi.

${ }^{9}$ Ferrari 2005: 683, n. 52. Sappiamo che Autolico era figlio di Hermes da una testimonianza post-omerica e, segnatamente, da Hesiod., fr. 64 M.-W. Da Omero stesso (Hom. Il. 11.266ss.) sappiamo che Autolico aveva derubato Amintore, figlio di Orcomeno, di un prezioso casco, decorato con zanne di cinghiale. Mi pare interessante anche riflettere sulla traduzione del

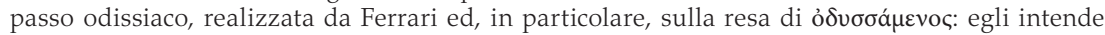
"covando odio", come a voler conferire al participio aoristo anche una sfumatura durativa che esso, di norma, non possiede, ma che addurrebbe - credo - un ulteriore argumentum, al fine di comprendere l'etimologia del nome di Odisseo. (In sostanza, Autolico odierebbe gli uomini al punto tale da voler lasciare traccia di questo odio, nel nome del nipote, destinato ad essere ricordato dai posteri.)

${ }^{10}$ In tal modo, Sofocle è, più volte, definito da Eustazio, sia nel commento all'Iliade che nel commento all'Odissea (Stallbaum 1925: $65 \kappa \tau \lambda$.) Per un approfondimento della questione, si veda Miller (1946). Del resto, nella stessa Vita 16 (Schultz 1835: 175) leggiamo: dicebat igitur [...] Sophoclem verum Homerum tragicum.

${ }^{11}$ Letteralmente: "sono eponimo dei mali".

${ }^{12}$ Ho cercato di proporre una mia traduzione per questo frammento sofocleo. Lloyd-Jones, nell'edizione dei frammenti sofoclei, curata per la Loeb, traduce così: "I am rightly called Odysseus, after something bad; for many enemies have been angry with me." (Lloyd-Jones 


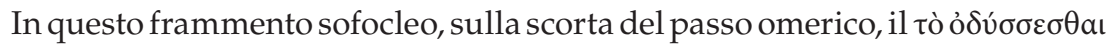
dà ancora sostanza all'ěm $\omega v v \mu$ ía. Tuttavia, diversa è la provenienza dell'odio o, se vogliamo, l'oggetto dell'odio. In tal caso, a prescindere dalla identificazione dei $\pi \mathrm{o} \lambda \lambda_{o}{ }_{1}^{13}{ }^{13} \mathrm{c}^{\prime}$ interessa il fatto che l'odio - che giustifica e, quasi, legittima il nome di Odisseo - è un odio diretto contro Odisseo stesso. Ciò è suggerito chiaramente da due elementi, entrambi posti in sedi privilegiate del trimetro: l'sìní, rafforzato dalla positio post caesuram, e l'žnoí, enfatizzato dall'esser collocato in explicit. Conformemente alla poetica sofoclea, che privilegia il singolo eroe, il singolo protagonista, Odisseo diviene egli stesso

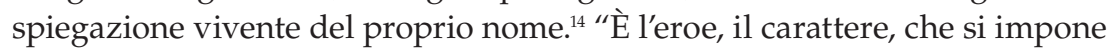
in Sofocle, che costituisce il centro delle sue tragedie; l'eroe come luogo di ciò che accade: referente costante dell'azione, delle parole del dramma."15

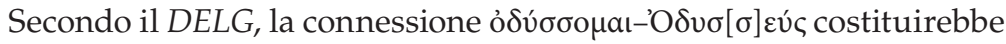
una "étymologie populaire",16 che rimonterebbe proprio ad Omero. Chantraine cita proprio Hom. Od.19.405 ss. come "le passage les plus esplicite",17 concludendo che "l'étymologie véritable est ignorée".18 $\mathrm{Ma}$, in questo contesto, più che la veridicità del procedimento di derivazione etimologica, $c^{\prime}$ in-

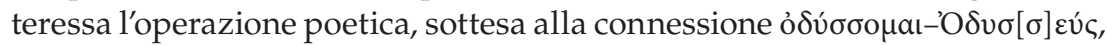
elaborata da Omero e mutuata cum variatione da Sofocle. Ma Sofocle, che non si dimostra mai un acritico e prosastico "riutilizzatore" dei fontes -

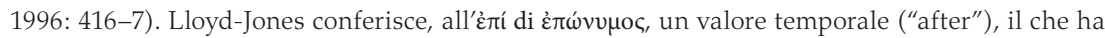
un chiaro valore esegetico, nel senso che tale traduzione suggerisce l'idea che Odisseo derivi il proprio nome dall'accadimento di eventi brutti. Tale interpretazione è sostenibile proprio se colleghiamo Soph. fr. 965 con Hom. Od. 19.405 ss., come, del resto, l'editore suggerisce - sia pur laconicamente - in nota. Io, pur essendo d'accordo con l'editore (del resto, il passo è citato proprio dalla Vita Sophoclis), ho cercato di proporre una traduzione un po' più neutra, tenendo conto anche del fatto che il frammento fa parte di quel gruppo di frammenti, per i quali non è stato possibile individuare una precisa tragedia di pertinenza. In ogni caso, è evidente la connessione

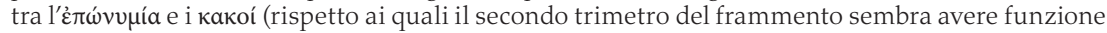
epesegetica). Infine, Lloyd-Jones intende $\pi$ o $\lambda \lambda$ oí ... $\delta v \sigma \mu \varepsilon v \varepsilon \tilde{c} \varsigma$ come un unico sintagma (aggettivo + aggettivo sostantivato), il che non è impossibile, considerato che l'iperbato è figura cara a Sofocle.

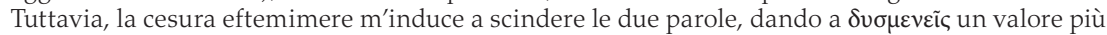
pregnante (quasi appositivo, rispetto a $\pi \mathrm{o} \lambda \lambda \mathrm{\lambda ó}_{\text {), }}$ valore che, in fondo, non fa che rafforzare il verbo

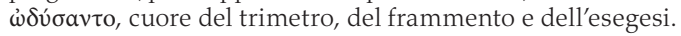

${ }^{13}$ S.v. la n. 10.

${ }^{14}$ Per quanto concerne la centralità dell'eroe sofocleo, s.v.: C. H. Withman, 1951, Sophocles: A Study of Heroic Humanism. Cambridge, Harvard University Press; G. Méautis, 1957, Sophocle. Essai sur le héros tragique, Parigi; G. M. Kirkwood, 1958, A Study of Sophoclean Drama, New York; B. M. W. Knox, 1964, The Heroic Temper, Berkeley-Los Angeles, University of California Press. Tra i lavori più recenti, s.v. : C. Miralles, 2009, La luce del dolore. Aspetti della poesia di Sofocle, Napoli, Liguori editore; A. Rodighiero, 2000, La parola, la morte, l'eroe. Aspetti di poetica sofoclea, Padova, Imprimitur.

${ }^{15}$ Miralles 2009: 12-13.

${ }_{16}$ Chantraine 1977: 776.

${ }^{17}$ Chantraine 1977: ibid.

${ }_{18}$ Chantraine 1977: ibid. 
mitici e/o poetici - compie un'ulteriore operazione poetica. A mio avviso, Sofocle opera, qui, una sorta di ri-etimologizzazione, rispetto al fons odissiaco: pur conservando l'etimologia, ne cambia l'angolazione, ne modifica il vettore - invertendolo - e, forse, proprio in questo mutamento di direzione è ravvisabile, in sostanza, lo scarto tra epos e tragedia.

Ma vi è un caso più evidente di ri-etimologizzazione sofoclea, per la cui analisi posso muovermi su un terreno meno scivoloso e sdrucciolevole di quello offerto dal fr. 965 appena analizzato: è il caso del nome proprio di Aiace, al quale Sofocle ha dedicato l'omonima tragedia - l'Aiace,,$^{19}$ appunto-, una delle sette pervenuteci integre. In questa tragedia, Aiace stesso riflette sul proprio nome e ne fornisce l'etimologia. ${ }^{20}$ Si consideri Soph. Aj. $430-3:{ }^{21}$

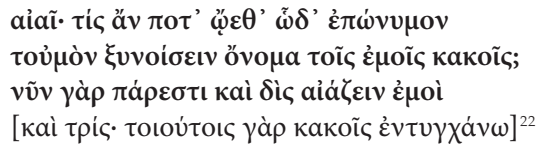

Aiai! Chi mai avrebbe pensato che il mio nome fosse così consonante alle mie sciagure? Ora posso ben gridare due [o tre volte 'Aiai': tali sono i mali in cui mi trovo.] (trad. di M. P. Pattoni) ${ }^{23}$

Questi versi sono inseriti nell'ampia j̃̃̃øৎ di Aiace, che coincide - secondo una prassi tragica non comune - con il secondo episodio. ${ }^{24}$ Dopo il

${ }^{19}$ Odisseo è uno dei protagonisti dell'Aiace. Tuttavia non ne costituisce l'eroe principale, né Sofocle qui si sofferma, in qualche modo, sull'etimologia del suo nome. Per tali motivi, non ho incluso riferimenti all'Aiace, per quanto concerne l'analisi del nome di Odisseo. Al contrario, Odisseo, nell'Aiace, rappresenta uno di quei personaggi "del tutto positivi, e in una certa misura 'vincenti' agli occhi del pubblico, perché costantemente versati nella realizzazione del giusto". (Rodighiero 2000: 168-9).

${ }^{20}$ Cfr. Fuochi 1898: 299.

${ }^{21}$ Per quanto concerne il testo sofocleo, mi avvalgo dell'edizione critica oxoniense, elaborata da Lloyd-Jones e Wilson (Lloyd-Jones et Wilson, 19901). Strumenti indispensabili per comprendere le ragioni delle scelte testuali più rilevanti sono: Lloyd-Jones et Wilson 1990² Lloyd-Jones et Wilson 1997. Per gli scholia, resta fondamentale l'edizione curata da Papageorgius 1888.

${ }^{22}$ Lloyd-Jones e Wilson, sulla scorta di Morstadt (1864: 43), espungono il v. 433, "for, without it, Ajax' grim pun, upon his name, is more effective" (Lloyd-Jones et Wilson 1990²: 19). Del resto, prima di Lloyd-Jones e Wilson, sempre sulla scorta del Morstadt, già Nauck e Fraenkel espungevano il trimetro, sentito come pleonastico. Kamerbeek, invece, difende il v. 433,

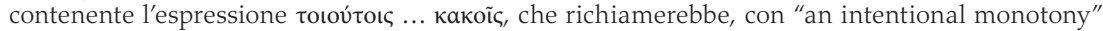

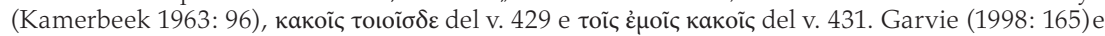
Finglass (2011: 264-5), invece, si pongono sulla medesima linea di Lloyd-Jones et Wilson et alii.

${ }^{23}$ Pattoni 1997: 153.

${ }^{24}$ Già lo scoliaste trovava rilevante la grande estensione della $\dot{\rho} \tilde{\sigma} \sigma \iota \varsigma$ di Aiace. S.v. schol. ad l.:

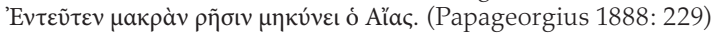


концо́ৎ, Aiace esprime il proprio dolore, "reflecting on the terrible appropriateness of his name": ${ }^{25}$ con piena consapevolezza intellettuale, recupera il concetto tipicamente greco, secondo cui esiste un preciso rapporto di

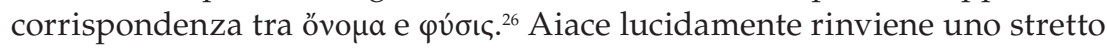
legame tra il suo dolore, espresso con la lamentosa interiezione aia $\tilde{i}^{27}$ - ed il suo nome. La riflessione che Aiace fa, in merito al proprio nome, sembra derivare "from the common Greek notion that a name is not a matter of convention, but belongs naturally to its bearer, and provides a clue to his character or destiny".28

Naturalmente esistevano già etimologie pre-sofoclee per il nome di Aiace, come la "more optimistic etimology"29 nelle Eoie esiodee - come ricorda Finglass - o come l'etimologia pindarica, secondo la sarebbe il nome dell'eroe sarebbe connesso con l'apparizione di un'aquila: Aíaç-ai̊tóc. Si consideri, a tal proposito Pind. Isthm. 5. 53:

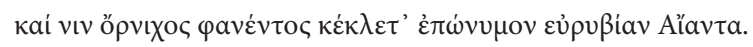

Ma quella sofoclea costituisce una vera e propria ri-etimologizzazione post eventum, se, per eventum, intendiamo la strage di bestiame che Aiace compie, durante la notte, perché reso folle da Atena, e che Sofocle presuppone come evento che, pur non rappresentato - ma fortemente alluso - dà l'avvio al dramma.

I vv. 430-2 - in particolare attraverso la dolorosa esclamazione aiai ed il verbo aiáčlı $v^{30}$ - richiamano un passo della ṕñoı di Tecmessa, nel lunghissimo, primo episodio, che può arricchire l'esegesi del passo appena esaminato; si tratta di Soph. Aj.317-2:

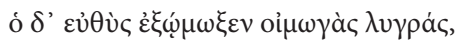

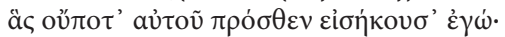

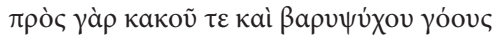

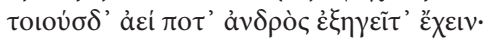

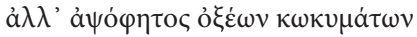

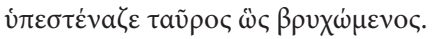

${ }^{25}$ Finglass 201: 264.

${ }^{26}$ Cfr. Griffith 1978: 84, n. 5.

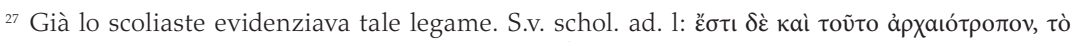

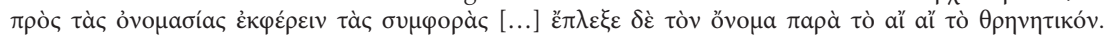
(Papageorgius 1888: 229)

${ }^{28}$ Garvie 1998: 165.

${ }^{29}$ Finglass 2011: 265.

${ }^{30}$ Aỉaĩ ed aíáčı costituiscono, appunto, i due elementi per mezzo dei quali Aiace fornisce esplicitamente l'etimologia del proprio nome. 
E subito proruppe in amari gemiti, quali prima non avevo mai udito da lui: infatti diceva sempre che tali lamenti sono propri soltanto di un uomo da nulla, un vile. Senza strepito di acute grida, gemeva sordamente, come un toro mugghiante. (Trad. di M. P. Pattoni) ${ }^{31}$

Come commenta Di Benedetto, "a proposito dell'esplosione di pianto, Tecmessa precisa che si tratta di un modo di piangere completamente diverso da come egli usava manifestare il suo dolore prima che la follia lo colpisse. Egli infatti evitava acuti gridi di dolore [...] e comprimeva i suoi lamenti mugghiando come un toro. Quando invece Aiace apprende dalla donna cosa sia effettivamente successo, esplode in un lamento incontrollato". ${ }^{32}$ Ciò significa che la strage che Aiace ha provocato ed il conseguente dolore, che egli ha patito, hanno causato, in lui, un profondo cambiamento: ciò lo conduce ad abbracciare una nuova forma comportamentale, che va a legittimare il suo lamento e che - mi sembra - può render anche conto anche del motivo per il quale Sofocle abbia atteso i vv. 430 ss per fornire l'etimologia del nome dell'eroe. Fornire l'etimologia dell'eroe prima non avrebbe avuto senso: era necessario, dapprima, che l'eroe fosse folle e che poi, rinsavito, prendesse coscienza della propria follia, attraverso le parole di Tecmessa. Pertanto, l'inserimento dell'etimologia del nome proprio, oltre ad essere rifunzionalizzata in relazione al contenuto della tragedia, risulta anche adatta all'economia del dramma.

Per completare il quadro, si consideri anche Soph. Aj.904:

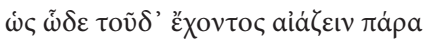

Se egli è in questa condizione, a noi non rimane che il pianto.

(Trad. di M. P. Pattoni) $^{33}$

Qui, nell'ambito del dialogo lirico col coro, ${ }^{34}$ Tecmessa attribuisce a se

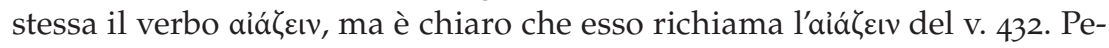
raltro - come ci conferma il ThLG - si tratta delle uniche occorrenze sofoclee di tale verbo. Il legame tra i due passi è confermato dal fatto che il coro, al v.

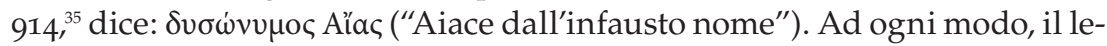

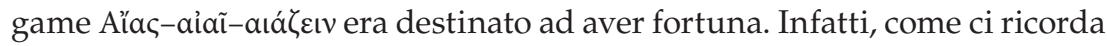
Jebb, "the association of Aila

${ }^{31}$ Pattoni 1997: 143.

${ }^{32}$ Di Benedetto 1983: 37.

${ }^{33}$ Pattoni 1997: 191.

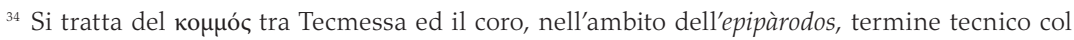
quale si suole definire il secondo ingresso del coro (fatto raro in tragedia).

${ }_{35}$ Tra l'altro, come nota Fuochi (1898: 299), il v. 914 costituirebbe una ripresa compendiata dell'etimologia del nome di Aiace. 
(on whose petals the letters AI were supposed to be legible) sprang from the blood of Ajax, as it had sprung from that of Hyacinthus". ${ }^{36}$ A tal proposito, si consideri Ov.Met.13.397-8:

Littera communis mediis pueroque viroque Inscripta est foliis; haec nominis illa querelae.

Una sigla è iscritta tra i petali, identica per il giovinetto e per l'eroe: per quest'ultimo [scil. per Aiace] si tratta delle iniziali del nome, per il primo del simbolo del lamento. (trad. di G. F. Villa) ${ }^{37}$

Vorrei concludere questa mia breve analisi, mutuando le parole di Miralles, il quale scrive:

Non c'è parola che non si compia o che non sembri viva nella tragedia di Sofocle. Anche i nomi propri, che a volte valgono solo come tali sino a che qualche verso, qualche gioco di parole, o il risultato stesso dell'azione, ${ }^{38}$ non li accenda e non li carichi di senso. ${ }^{39}$

\section{BIBLIOGRAFIA ESSENZIALE}

Allen, Th.W., ed. 1908. Homeri Opera. III-V. Oxford (1917-1919).

Chantraine, P. 1977. Dictionaire étymologique de la langue grecque. Histoire des mots. Paris: Les Éditions Klincksieck.

DELG $=$ Chantraine 1977.

Di Benedetto, V., ed. 1983. Sofocle. Firenze: "La nuova Italia" Editrice.

Di Benedetto, V., ed. 2010. Omero: Odissea. Milano: BUR.

Ellendt, H.; Genthe, H. 1872. Lexicon Sophocleum, vol. I-II. Berlin.

Ferrari, F. 2005. Omero: Odissea. Torino: UTET.

Finglass, P.J. 2011. Sophocles: Ajax. Cambridge-New York: Cambridge University Press. (Cambridge Classical Texts and Commentaries 48)

Fuochi, M. 1898. "Le etimologie dei nomi propri nei tragici greci." Studi Italiani di Filologia Classica 6: 273-318.

Garvie, A.F., ed. 1998. Sophocles. Ajax. With introduction, translation and commentary. Oxford: Aris \& Phillips Classical Texts.

Griffith, M. 1978. "Alkestis 636-641." Harvard Studies in Classical Philology 82: 83-6.

Jebb, R. C. 1883-1896. Sophocles: The plays and Fragments, I-VII. Cambridge.

\footnotetext{
${ }^{36}$ Jebb 1883: 227 (vol. I).

${ }^{37}$ Villa 2010: 763.

${ }^{38}$ È proprio questo il caso di Aiace, come ho cercato di dimostrare supra.

${ }^{39}$ Miralles 2009: 18.
} 
Kamerbeek, J. C. 1963-1984. The Plays of Sophocles. 7 vols. Leiden: E. J. Brill.

Kirkwood, G.M. 1958. A Study of Sophoclean Drama. New York: Cornell University Press.

Knox, B.M.W. 1964. The Heroic Temper. Berkeley-Los Angeles: University of California Press.

Lloyd-Jones, H., ed. 1996. Sophocles. Fragments. London-Cambridge: Harvard University Press. (Loeb Classical Library)

Lloyd-Jones, H.; Wilson, N. G., eds. 1990¹. Sophoclis Fabulae. Oxford: Oxford University Press.

Lloyd-Jones, H.; Wilson, N. G., eds. 1990². Sophoclea: studies on the text of Sophocles. Oxford: Oxford University Press.

Lloyd-Jones, H.; Wilson, N. G., 1997. Sophocles: Second thoughts. Göttingen: Vandenhoeck und Ruprecht. (Hypomnemata n. 100)

LSJ $=$ Liddel et Scott.

Liddel, H. G.; Scott, R. 1996. A Greek-English Lexicon. Revised and augmented throughout by Sir. H. S. Jones, with the assistance of R. McKenzie. With a revised supplement. Oxford: Claredon Press.

M.-W. = Merkelbach et West.

Merkelbach, R.; West, M.L., eds. 1967. Fragmenta Hesiodea. Oxford: Claredon Press.

Méautis, G. 1957. Sophocle. Essai sur le héros tragique. Paris: Albin Michel.

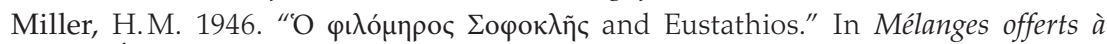
Édouard Delebecque, ed. C. Froidefond, 261-76. Aix-en-Provence: Université de Provence.

Miralles, C. 2009. La luce del dolore. Aspetti della poesia di Sofocle. Napoli: Liguori editore.

Miralles, C. 2012. "Il nome di Ulisse." Annali Online di Ferrara-Lettere 7.2: 1-11.

Morstadt, R. A. 1864. Beiträge zur Exegese und Kritik des sophokleischen Tragödie Elektra, Aias und Antigone. Schaffhausen.

Pattoni, M. P. 1997. Sofocle: Aiace, Elettra. Introduz. a cura di E. Medda. Milano: BUR.

Papageorgius, Petrus N., ed. 1888. Scholia in Sophoclis tragoedias vetera. Lipsiae: in Aedibus B. G. Teubneri.

$\mathrm{R}=$ Radt et Kannicht.

Radt, S.; Kannicht, R., ed. 1977. Tragicorum Graecorum Fragmenta, vol. IV (Sophocles). Göttingen.

Rodighiero, A. 2000. La parola, la morte, l'eroe. Aspetti di poetica sofoclea. Padova: Imprimitur.

Schneidewin, F.W.; Nauck, A. 1865. Sophokles, erklärt, vol. 1-3. Berlin.

Stallbaum, J. G., ed. 1925-1930. Eustathii Archiepiscopi Thessalonicensis Commentarii ad Homeri Odysseam. Lipsia.

Schultz, F. 1835. De vita Sophoclis poetae commentatio. Berlin: Phil. Diss.

Villa, G.F. 2010. Ovidio: Le metamorfosi. Note di R. Corti. Milano: BUR.

Withmann, C.H. 1951. Sophocles: A Study of Heroic Humanism. Cambridge: Harvard University Press. 
Resumo. Il contributo mira alla disamina di due nomi propri di eroi - segnatamente Odisseo ed Aiace -, all'interno della produzione sofocle. Nel contributo si cercherà di mostrare come Sofocle - ben consapevole dell'etimo invalso, fissato dal mythos e/o dalla precedente produzione lirica - abbia, in questi due casi particolari, attuato una consapevole ri-etimologizzazione, in funzione dello svolgimento del proprio dramma. Parole chiave. Sofocle, tragedia, Aiace, Odisseo, ri-etimologizzazione. 